\title{
Comparative Evaluation of the Shear Bond Strength of a Total Etch Adhesive with a Self-Etching Primer on an Endodontically Treated Teeth
}

\author{
Shamina Bawa, Mithra N Hegde
}

\begin{abstract}
The present in vitro investigation evaluated the shear bond strength of endodontically treated teeth restored with composite resin in conjunction with a total etch adhesive excite and a selfetching primer, Prompt-L-Pop.

The aim of this study was to evaluate and compare the bond strength of two generation bonding systems when used on endodontically treated teeth.

Twenty-four freshly extracted noncarious intact human maxillary and mandibular teeth were selected for this study. They were divided into 2 groups of 12 teeth each. The occlusal surface of the teeth was ground to prepare flat dentin surface. In group I, 5th generation dentin bonding system excite was used and in group 2, P rompt-L-pop, a self-etching primer was used. Both the dentin bonding agents were placed on the flat dentinal surfaces according to the manufacturer's instructions. Cylinders of composite resin were built on the prepared surface using Teflon mold. The prepared specimen were then embedded on an acrylic jig of suitable dimension and transferred to Instron machine for shear bond strength analys is. Shear bond strengths were measured at a cross head speed of $0.03 \mathrm{~mm} / \mathrm{sec}$.
\end{abstract}

Keywords: Total etch adhesive, Self-etching primer, Shear bond, Pulpal dentin.

How to cite this article: Bawa S, Hegde MN. Comparative Evaluation of the Shear Bond Strength of a Total Etch Adhesive with a Self-Etching Primer on an Endodontically Treated Teeth. World J Dent 2012;3(3):247-249.

\section{Source of support $\mathrm{Nil}$}

Conflict of interest: None declared

\section{INTRODUCTION}

Present day dentistry is unimaginable without adhesives. Composite resins is widely used and accepted for the treatment in almost all situations. Several clinicians in general practice prefer to use composite resins for all sort of clinical situations including postendodontic restoration. The concept of bonding a restorative material to dentinal surface is by no means a new idea. Even at the time of Buonocore using phosphoric acid to bond enamel, the idea of bonding to dentin was considered. ${ }^{1}$ The dentin bonding systems have been developed over the years and have become of particular interest to the success of tooth colored restorative materials that include ceramics, composites and compomers. With the introduction of newer generation bonding agents, the problem of technique sensitivity has reduced. $^{2}$
A resin composite restoration enables nonvital teeth to be restored by replacing only the lost tooth structure because the dentin bonding system can reinforce the remaining tooth structure. ${ }^{3}$ The successful use of dental adhesive materials relies on their proper selection and on understanding their properties, in particular their curing mechanism and application technique.

Enamel adhesion by means of acid etching has become an accepted technique in restorative dentistry. A dhesion to dentin, however is still under investigation. At this time, two distinct adhesive techniques are prominent in the attempt to establish a strong bond to dentin: Total etch and self-etching primers. ${ }^{4}$

I solated studies have indicated that chemical irrigants do adversely affect the bond strength of resin composite to dentin stored after endodontic treatment. ${ }^{5}$ Limited information exists covering dentin bonding to nonvital teeth in general and pulpal dentin in particular. Research on bond strength values against endodontically treated teeth is scarce. The aim of this study was to evaluate the effect of endodontic treatment on performance of bonding to pulpal dentin using two different dentin bonding systems.

\section{MATERIALS AND METHODS}

Twenty-four freshly extracted noncarious intact human maxillary and mandibular teeth were selected for this study. The occlusal surface of the teeth were ground on a water cooled trimming wheel to prepare flat dentin surface. Pul pal dentin is the dentin obtained after removal of the roof of the pulp chamber. They were divided into 2 groups of 12 teeth each. Group 1 was bonded using 5th generation total etch adhesive namely Excite. Group II was bonded using 1 step self-etching primer namely Prompt -L-Pop.

In group I, where excite was used, the prepared flat dentinal surface was etched using 35\% phosphoric acid for 15 seconds. It was then cleansed and dried using oil free water spray. Dentin bonding agent was then applied and cured according to the manufacturers instruction. This was followed by packing of composite resin over the prepared dentinal surface using Teflon mold measuring $3 \times 3 \mathrm{~mm}$ and cured layer by layer. 
In group II, one step self-etching primer Prompt-L-Pop was used. Here the liquid from the red blister was transferred into the yellow blister which was then transferred into the green blister which has the applicator tip. Using this applicator tip, the bonding agent was applied to the prepared dentinal surface with moderate finger pressure stream of air was used to evenly disperse the material into thin film. The material was then cured for 20 seconds. Composite resin was packed over this prepared surface using Teflon mold and cured layer by layer.

The prepared specimens were mounted on the acrylic jig of specific dimension and was transferred to the instron machine individually and subjected to shear bond strength analysis at a cross head speed of $0.3 \mathrm{~mm} / \mathrm{sec}$.

\section{RESULTS}

The present in vitro investigation evaluated the shear bond strength of Excite, a total etch adhesive in comparison with Prompt-L - Pop, a single step self-etching primer when used on pulpal floor dentin restored with single composite resin Z 100.

A ccording to this present study, Excite required the highest mean shear load to fracture [14.7 M Pa] when compared to Prompt-L-Pop [13.55 M Pa].

Group comparison was done using B oneferonni t-test. Highly significant results were observed when B oneferonni t-test was applied to the results.

\section{DISCUSSION}

In the present investigation, we have made an attempt to evaluate a globally accepted total etch adhesive, Excite, in comparison with more recently introduced self-etching primer Prompt-L-Pop.

Conventional three step dentin bonding system have been shown to provide reliable bonding. But the elaborate three steps required gives rise to possible problems through contamination of the bonded surface prior to the placement of resin composite restorative material. The problem of this technique sensitivity seems to have been reduced with the new generation dentin bonding systems. ${ }^{2}$

Conventional endodontic therapy begins with access cavity preparation and ends with obturation. The access cavity when conserved with minimum involvement, the usual choice as access restorative material is composite resins. The utilization of resins as access restorative material is almost routine in clinical practice.

Variation in dentin depth and permeability can significantly influence the bond strengths of direct resinbased restorative systems. ${ }^{6}$ Regional structural differences, such as caries affected dentin; sclerotic dentin and root dentin are important factors that can affect bond strengths to dentin. ${ }^{7}$ However, there is little information about the bonding performance of bonding systems to floor of pulp chamber dentin. Few studies have been conducted to evaluate the sealing capabilities of resins against enamel and coronal dentin while little research has been done on adhesion of resins to the floor of the pulp chamber dentin.

If one considers characteristics of the endodontically treated tooth, one should accept that the endodontic treatment performed, changes the actual composition of the remaining tooth structure. The tooth structure that remains after endodontic treatment is undermined and weakened by caries, fracture, tooth preparation and restoration. Endodontic access into the pulp chamber destroys the structural integrity provided by the coronal dentin of the pulpal roof and allows for greater flexing of the tooth under function. ${ }^{8}$

Nikai do et al ${ }^{9}$ discussing bonding to nonvital teeth are of theopinion that the decreased strength seen in endodontically treated teeth is primarily dueto loss of coronal tooth structure and is not a direct result of endodontic treatment.

A kagawa $\mathrm{H}$ et al ${ }^{10}$ observed that the different region of superficial, deep and pulp chamber dentin demonstrated different morphological characteristics. The ratio of dentin tubules to inter tubular dentin in deep dentin was more than that of superficial or pulpal floor dentin, where as diameter of tubules in pulpal floor dentin was smaller than that of coronal dentin. These morphological differences according to A kagawa et al will have an effect on the conductance of fluid within dentin and thereby on the bond strength. However, the bond to radicular and pulp chamber dentin does seem to vary quite a lot depending on the dentin bonding agent used. ${ }^{11}$ Hence, it is essential to give importance to the selection of dentin bonding agent used for these regions of the tooth.

When it comes to dentin bonding systems, it is important to follow the manufacturer's instructions carefully. Over etching can create a region of poorly or uninfil trated dentin. This zone may be susceptible to acid or enzyme attack from oral bacteria, hence leading to bond failure. ${ }^{12}$

In the present investigation, comparatively lower bond strength values were recorded in comparison to previous studies with both the bonding systems tested. Reason for this lower value may be because most of the teeth used for the study were from older age group, periodontal involved and subjected to various masticatory loading during the life of the tooth. Further to this, dentin as a bonding substrate is heterogeneous. The surface wetness, dentin permeability are all nonuniform on the flat surface of dentin. There is high tubule density in the pulp chamber level and hence 
Comparative Evaluation of the Shear Bond Strength of a Total Etch Adhesive with a Self-Etching Primer on an Endodontically Treated Teeth

little intertubular dentin between tubules to permit hybrid layer formation. ${ }^{13}$

Seema B elli et $\mathrm{al}^{14}$ in their study on regional strengths to adhesive resin to pulp chamber dentin suggested that clinicians should choose an adhesive system that is simple, easily retrievable and technique insensitive.

W ith the introduction of self-etching primers and newer adhesive resin system and bonding techniques it is hoped that in future these can be adapted for use within pulp chamber to provide second layer of defense against microleakage and at the same time have sufficient bond strength values to pulpal dentin, so that there is no scope of debonding of access restoratives.

\section{CONCLUSION}

This investigation concluded that the total etch adhesive Excite (Group I) fractured at the highest mean shear load while Prompt-L -Pop, a 1 step self-etching primer (G roup II) fractured at the lowest value when used on endodontically treated teeth. Highly significant results w ere observed when Boneferonni t-test was applied to the results.

The purpose of this study was to evaluate the dentin shear bond strength of two adhesive systems on an endodontically treated teeth. It may be concluded that the shear bond strength of dentin is dependent on material (adhesive system), substrate depth and adhesive/depth interaction.

Major developments in adhesive materials, especially dentin bonding systems are seen in the past years. Further improvements in the reliability and ease of use of DBA can make restoration of endodontically treated teeth easier and stronger.

\section{REFERENCES}

1. Tyas M J, B urrow M F. A dhesive restorative materials: A review. A ustralian Dent J our 2004;49(3):112-21.

2. Sano $\mathrm{H}, \mathrm{K}$ anemura N, Burrow M F, et al. Effect of operator variability on dentin adhesion: Students vs dentists. D ent $M$ ater J 1998;17:51-58
3. Burrow M F, Sano H, Nakajima M , et al. B ond strength to crown and root dentin. A m J Dent 1996;9:223-29.

4. Kijsamanmith K, Timpawat S, et al. M icrotensile bond strengths of bonding agents to pulpal floor dentin. Int Endod J 2002;35:833-39.

5. W ang $Y$, Spencer $P$. Hybridization efficiency of the adhesive/ dentin interface with wet bonding. J Dent Res 2003;30:99-105.

6. Y oshiyama M , U rayama A, M atsuo T. Bonding to pulpal floor dentin. $M$ odern trends in adhesive dentistry proceedings of the adhesive dentistry forum. Y okohama, Japan 1999;91-95.

7. Shono $Y$, Ogawa T. Regional measurement of resin dentin bonding as a array. Journal Dental Reaserch 1999;78(2): 699-705.

8. Yoshikawa T, Sano H, Burrow MF, Tagami J, Pahley DH. Effects of dentin depth and cavity configuration on bond strength. J Dent Res 1999;78(4):898-905.

9. Nikaido T, Takano Y, Sasafuchi $Y$. B ond strengths to endodontically treated teeth. A m J Dent 1999 A ug;12(4):177-80.

10. A kagawa Hirotoshi, Toru Nikaido, Tsunehiko Takada. Shear bond strengths to coronal and pulp chamber floor dentin. A m J Dent 2000;15(6):383-88.

11. Jorge Perdigao, Ronald F, B runo T. N ew trends in dentin/enamel adhesion. A m J Dent 2000;13:250-300.

12. M acro Ferrari, Francesco M annocci. Bonding to root canal: Structural characteristics of the substrate. A m J Dent 2000;23:481-86.

13. Ogata $M$, Okuda $M, N$ akajima $M$. Influence of the direction of tubules on bond strength to dentin. Operative Dentistry 2001:26:27-35

14. Seema B elli, Y Zhang, Patrica N. Regional bond strengths of adhesive resins to pulp chamber dentin. JN Endodon 2001;27(8):527-32.

\section{ABOUT THE AUTHORS}

\section{Shamina Bawa (Corresponding Author)}

Reader, Department of Cons and Endodontics, DAPM RV Dental College, 24th Main, First Phase, JP Nagar, B engaluru-78 Karnataka, India, Phone: 99801 00839, Fax: 080-26658411 e-mail: shammibawa@ rediffmail.com

\section{Mithra N Hegde}

Professor and $\mathrm{H}$ ead, D epartment of Cons and Endodontics, A B Shetty M emorial Institute of Dental Sciences, M angal ore, K arnataka, India 